\title{
Regionale Potenziale für die Bereitstellung von Energieholz - Ergebnisse einer Studie in Nord-Ost Brandenburg
}

\author{
Mareike Schultze, Marco Hahs, Stefan Siegemund
}

\section{Zusammenfassung}

Die dezentrale Bereitstellung und Nutzung von Energie aus holzartiger Biomasse kann sowohl zu Klimaschutzzielen als auch zur wirtschaftlichen Entwicklung ländlicher Gebiete beitragen. Im Projekt belo-net [1] werden anhand der Region Nord-Ost-Brandenburg Ansatzpunkte für Kooperationen in regionalen Wertschöpfungsketten mit Schwerpunkt auf dem Bereich der Energieholzlogistik untersucht. Zur Bildung geographischer Handlungsschwerpunkte werden eine Rohstoff- und eine Infrastrukturanalyse kombiniert. Im Ergebnis können mögliche Standorte für Biomassehöfe, die als Lager-, Umschlagund Verteilzentren wesentliche logistische Elemente regionaler Energieholzstrategien sind, identifiziert und bewertet werden. Waldenergieholz könnte in Nord-OstBrandenburg je nach Mobilisierung und Nutzung der vorhandenen Rohstoffpotenziale $5-6 \%$ des regionalen Wärmebedarfs decken.

\section{Abstract}

Sustainable production of energy from forest biomass helps protecting the climate and contributes to a balanced economic development in rural areas. In the northeast of Brandenburg the conditions for bio-energy production are very good. Important means to gain access to unused biomass resources, especially in the private forests, are the cooperation of companies in regional value chains and - as logistical hubs - regional storage and distribution centres. This paper introduces a method using geographical information systems (G.I.S.) to identify areas with sufficient resources for developing value chains and setting up storage and distribution centres. In the first step, we modelled the geographical distribution of forest biomass potentials based on planning data of the forest administration. By combining these data with an analysis of infrastructure for transport and storage, we could identify more than 40 suitable sites for storage and distribution centres. If mobilisation projects take effect forest biomass could contribute a share of 5 $6 \%$ to the heat supply in northeastern Brandenburg.

\section{Einleitung}

Das Land Brandenburg will bis zum Jahr 2020 seinen $\mathrm{CO}_{2}$ Ausstoß gegenüber dem Jahr 1990 um 40 \% senken (Landesregierung Brandenburg 2006). Hierfür soll der Bereich der erneuerbaren Energien unter Nutzung der in Brandenburg nachhaltig vorhandenen Potenziale massiv ausgebaut werden. Biomasse, darunter auch Energieholz, soll mit einem Anteil von über $40 \%$ an den erneuerbaren Energien im Jahr 2020 eine bedeutende Rolle spielen.

Die Erzeugung und Nutzung von Holzenergie kann neben positiven Umweltwirkungen auch einen wesentlichen Beitrag zur Stärkung der Wirtschaftskraft in ländlichen Gebieten leisten. Um dies zu erreichen, müssen vorhandene endogene Potenziale - wie z. B. Biomasserohstoffe, Arbeitskräfte, Infrastruktur und Know-how - aktiviert werden (Hoffmann 2007) und ein möglichst hoher Teil der Wertschöpfung im regionalen Kontext erzeugt werden (Gothe/Hahne 2005). Der Aufbau von Kooperationen zwischen regionalen Akteuren in Wertschöpfungsketten wird in diesem Zusammenhang ein wichtiges Instrument der Regionalentwicklung gesehen (Schubert 2007).

Im Rahmen des Projekts belo-net werden Ansatzpunkte für solche Kooperationen am Beispiel einer Projektregion erarbeitet. In einem ersten Schritt werden regionale Schwerpunkte identifiziert, an denen gute Voraussetzungen für den Aufbau von Produkt- und Dienstleistungsketten mit hoher regionaler Wertschöp- 
fung im Bereich Holzenergie bestehen. Als Einflussfaktoren werden die verfügbaren Rohstoffmengen und die vorhandene Infrastruktur für den Aufbau von Biomassehöfen herangezogen. Biomassehöfe sind als Lager-, Umschlag- und Verteilzentren wesentliche logistische Elemente regionaler Energieholz-Strategien. Unter Nutzung von Planungs- und Geodaten wurde die geographische Verteilung vorhandener Rohstoff- und Infrastrukturpotenziale analysiert. Die Ergebnisse wurden in einem »G.I.S. Energieholz[2]« zu einer Bewertungsbasis zusammengeführt.

\section{Die Untersuchungsregion Nord-Ost-Brandenburg}

Das Projektgebiet umfasst die Landkreise MärkischOderland, Barnim, Uckermark und Oberhavel. Mit einer Waldfläche von rd. 265.000 ha und einer recht geringen Besiedlungsdichte von 94 Einwohnern $/ \mathrm{km}^{2}$ (Statistik Berlin Brandenburg 2010a) bieten sich gute Möglichkeiten der Energieversorgung mit Holz. Innerhalb des Gebiets bestehen allerdings starke regionale Unterschiede mit landwirtschaftlich geprägten Gebieten in der Uckermark und Märkisch-Oderland (Bewaldungsgrad 22,9\% bzw. 24,4\%), und dem waldreichen Barnim (46,4 \% Wald) (Statistik Berlin Brandenburg 2009). Darüber hinaus entstehen durch den ausgeprägten Stadt-Land-Gradienten zwischen den berlinnahen und den peripheren Gebieten unterschiedliche Möglichkeiten zum Einsatz von Holz als Energieträger. So eignen sich dicht besiedelte Gegenden eher zur Versorgung von Haushalten mit Strom und Fernwärme, die in größeren Biomasseheiz(kraft)werken erzeugt werden. Für einzelstehende Häuser mit ausreichend Lagerfläche kommen besonders in Waldnähe beispielsweise moderne Stückholzanlagen in Frage.

Im Nord-Ost-Brandenburg produzieren allein die fünf Biomasseheizkraftwerke jährlich Strom im Wert von rd. 18 Mio. € (Vergütung nach EEG, Schneider \& Aust 2010), Wärme mit einem geschätzten Wert von 37 Mio. € (Wärmepreis nach BMWI 2010) und schaffen direkt etwa 20 Vollzeitstellen (Personalschlüssel gemäß FNR 2005). Hinzu kommen die Wärmeproduktion in Kleinanlagen sowie Umsätze und Beschäftigte in den Bereichen Forstwirtschaft und Bereitstellungslogistik.

Derzeit können rund 140 Betriebe Tätigkeitsbereichen zugeordnet werden, die mit der Bereitstellung von Energieholz zusammenhängen. Im Bereich der Logistik profitieren von einem weiteren Wachstum des Energieholzsektors nicht nur die rund 80 in der Region ansässigen Forstdienstleister sondern auch landwirtschaftliche Lohnunternehmer, Holzhandelsunternehmer und Transporteure.

Im Bereich der energetisch nutzbaren Holzrohstoffe wurden im Jahr 2009 ca. 20 Tsd. t atro[3] Altholz erfasst (LUGV 2010a; LUGV 2010b, Thraen/Kostmann 1997; Öko-Institut 2004). Eine Befragung der verantwortlichen Stellen im Landkreis Märkisch-Oderland und eine Hochrechnung mittels Geodaten ergaben ein jährliches Holzaufkommen von etwa $5.000-6.000 \mathrm{t}$ atro aus der Pflege von Grünflächen und Straßenrändern. Davon fallen in Märkisch-Oderland 70 \% im Verantwortungsbereich der Bau- und Grünflächenämter der Ämter und amtfreien Gemeinden an, 25 \% im Landesbetrieb Straßenwesen Brandenburg und der Rest unter Verantwortung der Kreisstraßenmeisterei des Landkreises Märkisch-Oderland.

Eine weitere noch im Anfangsstadium befindliche Form der Produktion von Energieholz ist der Anbau schnellwachsender Baumarten wie Pappel, Weide oder Robinie auf landwirtschaftlichen Flächen. Vor allem in den ausgeräumten Landschaften der Uckermark und im Osten Märkisch-Oderlands können Agrarholzflächen zum Strukturreichtum beitragen sowie Erosionsschutz- und Biotopfunktion haben. Murn (2008) weist Vorzugsflächen für die Agrarholzproduktion in der Region aus, auf denen sich theoretisch jährlich rund 210 Tsd. $t$ atro Agrarholz produzieren ließen.

Die heute weit bedeutendste Quelle für Energieholz ist die forstwirtschaftliche Produktion. Bei Aktivierung der ungenutzten Ressourcen im Bereich des Waldenergieholzes bestehen noch erhebliche Entwicklungsmöglichkeiten. Durch den dezentralen Anfall und die Bedeutung persönlicher Kontakte - gerade bei der Mobilisierung von Holz aus dem Privatwald[4] - bietet Waldenergieholz gute Möglichkeiten für weitere Anlagen im unteren Leistungsbereich und für den Betrieb privater Holzheizungen.

\section{Potenziale an Waldenergieholz}

Die Bestands- und Besitzstrukturen in den verschiedenen Teilregionen Nord-Ost-Brandenburgs bieten unterschiedlichste Voraussetzungen für die Bereitstellung von Waldenergieholz. Deshalb wurden die verfügbaren Rohholzmengen basierend auf den Forstplanungsdaten 


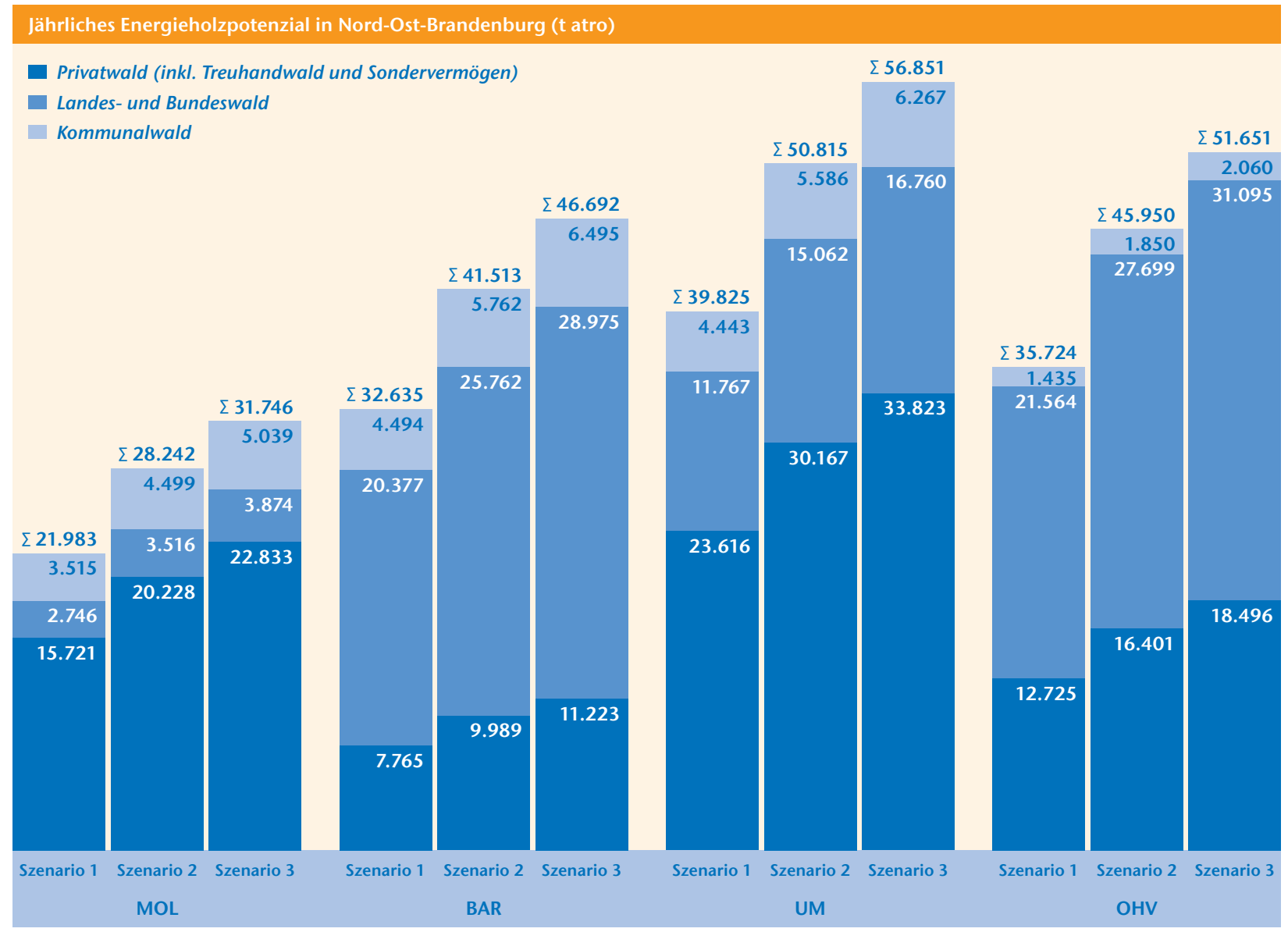

Abb. 1: Energieholzpotenzial nach Landkreisen, Waldbesitzarten und Szenarien in Nord-Ost-Brandenburg (t atro)

von Einzelbeständen flächenscharf modelliert. Die Datengrundlage besteht aus der digitalen Forstgrundkarte (FGK) und dem Datenspeicher Wald (DSW 2 Stand 2006), einer Datenbank mit detaillierten Struktur- und Sachdaten für jeden Bestand. Auf dieser Basis wurde der laufende jährliche Zuwachs getrennt nach verschiedenen Baumartengruppen[5] und deren Anteil an der Waldfläche ermittelt.

Waldenergieholz fällt bei der Pflege von Jungbeständen in Form von Vollbäumen und in älteren Beständen (Durchforstungsmaßnahmen oder Endnutzung) zusätzlich zu den stofflichen Rohholzsortimenten als sogenanntes Koppelprodukt an. Energetisch nutzbare Baumteile sind in letztem Fall Kronenholz, Reisig und unverwertbares Derbholz (Hepperle 2007).

DieModellierungdes Potenzials an Energieholzerfolgt verwendungsorientiert mithilfe von Bestandessortentafeln (Offer/Staupendahl 2009), die der Marktaktualität angepasst wurden. Neben den stofflichen Sortimenten Stammholz und Industrieholz werden Energieholz und nicht verwertbares Derbholz (NVD) unterschieden. Die Anteile an Astholz sind entsprechend der jeweiligen Höhe der Bäume als Mittelwerte nach Haschke und Ri- ckert (2005) berücksichtigt. Um den Einfluss verschiedener Holzvermarktungsziele darzustellen, wurden drei Varianten der Sortimentsbildung verglichen:

Szenario 1: Das Derbholz wird in Stammholz und Industrieholz unterteilt, die stoffliche Nutzung des Rohholzes steht im Vordergrund. Das Energieholzpotenzial besteht aus dem ermittelten Energieholzanteil und $60 \%$ des NVD

Szenario 2: Das Energieholzpotenzial besteht aus dem ermittelten Energieholzanteil und $80 \%$ des NVD

Szenario 3: Das Energieholzpotenzial besteht aus dem ermittelten Energieholzanteil und $80 \%$ des NVD. Darüber hinaus werden die Konkurrenz zwischen stofflicher und energetischer Nutzung berücksichtig und 10\% des Industrieholzanteils dem Energieholzpotenzial zugeschlagen.

Entlang der Erntekette entstehen an verschiedenen Stellen stoffliche Verluste. Die Umrechnung von Vorratsfestern in Erntefestmeter erfolgt mittels der im DSW 2 befindlichen Umrechnungsfaktoren in Abhängigkeit 
des Brusthöhendurchmessers. Verluste bei Ernte, Aufarbeitung und Rückung werden pauschal mit $15 \%$ berücksichtigt (Becker/Engler 2009).

Für die Waldfläche Nord-Ost-Brandenburgs lässt sich inklusive NVD ein technisches Energieholzpotenzial von jährlich rund 220 Tsd. $t$ atro ausweisen. Die erschließbare Menge wurde über die drei Szenarien im Bereich zwischen 100 und 170 Tsd. t atro eingegrenzt. Die Verteilung auf Landkreise und Waldbesitzarten zeigt Abbildung 1. Entsprechend des Planungshorizonts der zugrunde liegenden Forsteinrichtungsdaten sind die ermittelten Ergebnisse für einen Zeitraum von 10 Jahren aussagekräftig.

\section{Infrastruktur für Biomassehöfe}

Biomassehöfe können als Mittler zwischen Biomasseproduzenten und -abnehmern eine entscheidende Rolle für die Entwicklung des Energieholzsektors spielen. Sie bieten die infrastrukturelle Grundlage für die Bündlung von Mengen, für die Vorratshaltung und für die Aufbereitung von Ausgangsmaterialien unterschiedlicher Qualität. Damit helfen sie, bisher ungenutzte Kleinmengen aus dem Privatwald und der Landschaftspflege zu mobilisieren und $\mathrm{zu}$ vermarkten. Mit verschiedenen Veredelungsprozessen können an Biomassehöfen hochwertige Brennstoffprodukte hergestellt werden, die deutlich höhere Preise als unaufbereitetes Energieholz erzielen. Mittels sachgerechter Lagerung und rascher Trocknung des erntefrischen Materials (z. B. durch Nutzung der Abwärme von Biogasanlagen) werden außerdem Massenverluste vermieden[6].

Durch Biomassehöfe entstehen in ländlichen Gebieten zusätzliche Möglichkeiten der Wertschöpfung und Arbeitsplätze auch für gering Qualifizierte. Regionale Unternehmen der Land- und Forstwirtschaft und des Logistiksektors profitieren von zusätzlichen Aufträgen und geringen Transaktionskosten.

GeeigneteStandorte für Biomassehöfe haben eine gute Anbindung an die Versorgungs- und Verkehrsinfrastruktur und liegen in räumlicher Nähe zu Rohstoff- und Absatzmärkten. Idealerweise sind bereits für die Lagerung geeignete Flächen oder Gebäude vorhanden. Ebenfalls zu beachten sind die rechtlichen Rahmenbedingungen für den Betrieb der Höfe. Die konkrete Auswahl von Flächen zum Aufbau von Biomassehöfen erfordert detaillierte Vor-Ort-Analysen unter Einbeziehung der Akteure, die am Bewilligungsverfahren und am Betrieb des Hofes beteiligt oder davon betroffen sind. Eine Vorauswahl grundsätzlich geeigneter Standorte ist jedoch mittels G.I.S. möglich (vgl. auch Kühmaier et al. 2007). Unter Verwendung des Digitalen Landschaftsmodells Brandenburg (Version 2009) wurden mögliche Standorte in der Untersuchungsregion anhand von Ausschluss- und Eignungskriterien eingegrenzt. Ausgeschlossen wurden

v Flächen in bestehender Nutzung (Flugplätze, Talsperren/Wehre; Tagebau/Rieselfeld/Schwimmbecken; Grünland/Gartenland/Moor; Waldflächen, Ackerflächen; Sonderkulturanbau; Flächen besonderer funktionaler Prägung)

- Naturschutzgebiete

- Geschützte Bereiche des menschlichen Wohnens/ Lebens (Freizeitanlagen, Wohnbaufläche, Flächen im Abstand < 250 m um Wohnbauflächen)

- Geschützte Bereiche von übergeordneter Bedeutung (Wasserschutzgebiete I - III; Truppenübungsplätze)

- Als bau- oder betriebsuntauglich einzustufende Flächen (Seen, Flüsse, Sumpf/nasser Boden; Inseln) sowie ein Puffer von $30 \mathrm{~m}$ um Waldflächen

- Flächen in Industriegebieten, die jedoch für den Betrieb von Lagerplätzen als ungeeignet einzustufen sind (z. B. Einhandelsflächen).

Als Eignungskriterien für Biomassehöfe wurden die Verkehrsanbindung, die vorhandene Betriebsinfrastruktur (bzw. schnelle und kostengünstige Anschlussmöglichkeiten) und die Verfügbarkeit überschüssiger Energie zur Trocknung des Holzes verwendet. Für diese Kriterien wurden Attribute festgelegt, die durch Zuordnung von Bewertungspunkten zu einer Vergleichsbasis zusammengeführt wurden:

- Verkehrsanbindung: Euklidischer Abstand zu Autobahnen, Bundes- und Landstraßen (1, 3 und $5 \mathrm{~km}$ ).

- Betriebsinfrastruktur: Industriegebiete der Nutzungsvarianten Lagerflächen, Anlagen und Gebäude zur Verwertung und Entsorgung von Abwasser und Abfallstoffen, Anlagen und Flächen zur Versorgung der Allgemeinheit mit Elektrizität, Wärme, Wasser und andere Flächen.

- Energie zur Trocknung des Holzes: Bewertung der Standorte in Umkreisen von 200, 500 und $1.000 \mathrm{~m}$ zu den aktuell im Untersuchungsgebiet in Betrieb befindlichen Biogasanlagen gemäß HNE (2010).

Alle grundsätzlich geeigneten Flächen wurden nach dem Verschneiden der bewerteten Einzellayer in Eignungsklassen eingeteilt. Abbildung 2 zeigt die geographische Verteilung der Standorte, die als "gut« oder »sehr gut« geeignet bewertet wurden. 

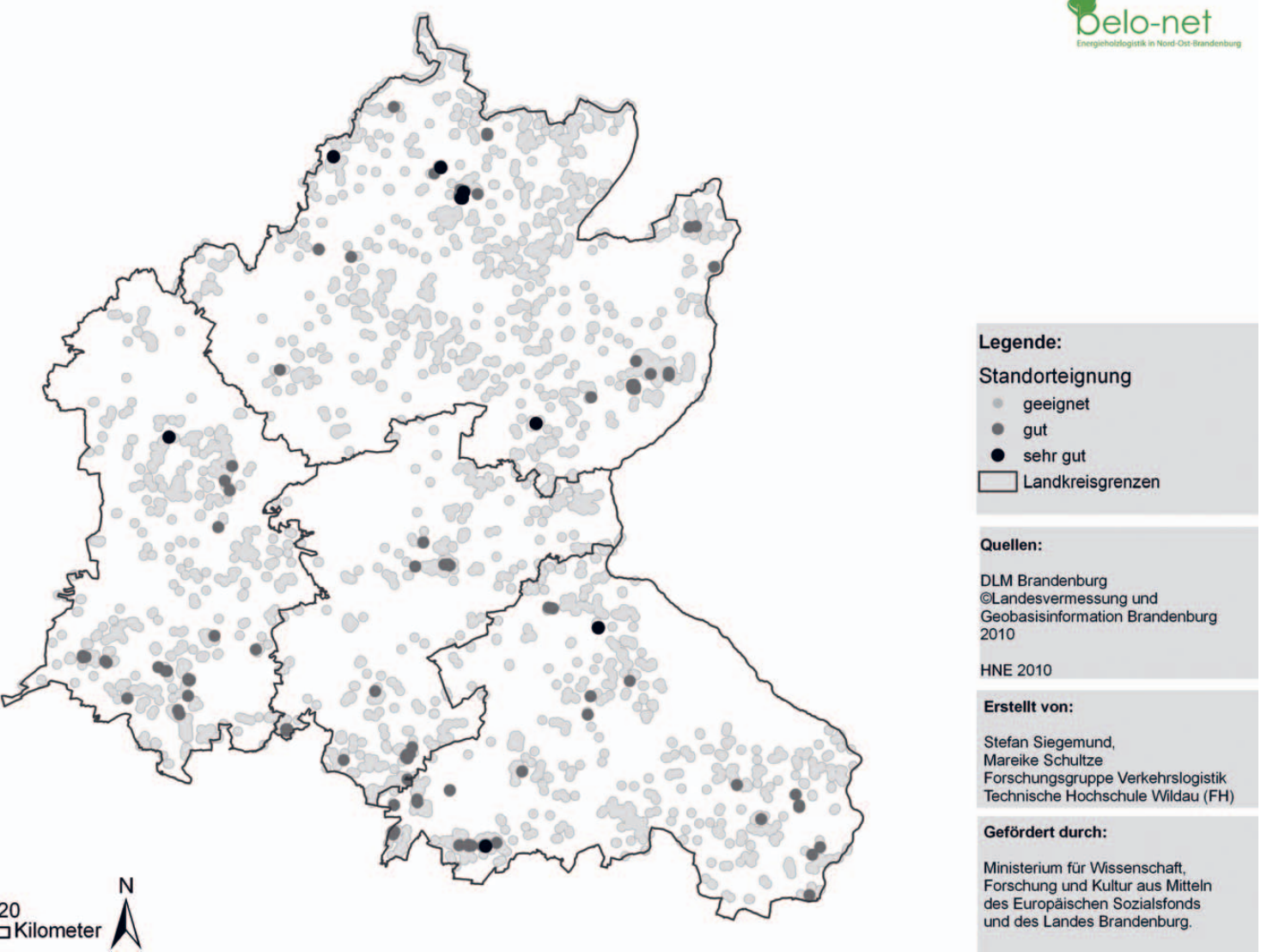

Abb. 2: Standorte in Nord-Ost-Brandenburg, die nach den verwendeten Ausschluss- und Eignungskriterien als für den Aufbau von Biomassehöfen geeignet gelten können

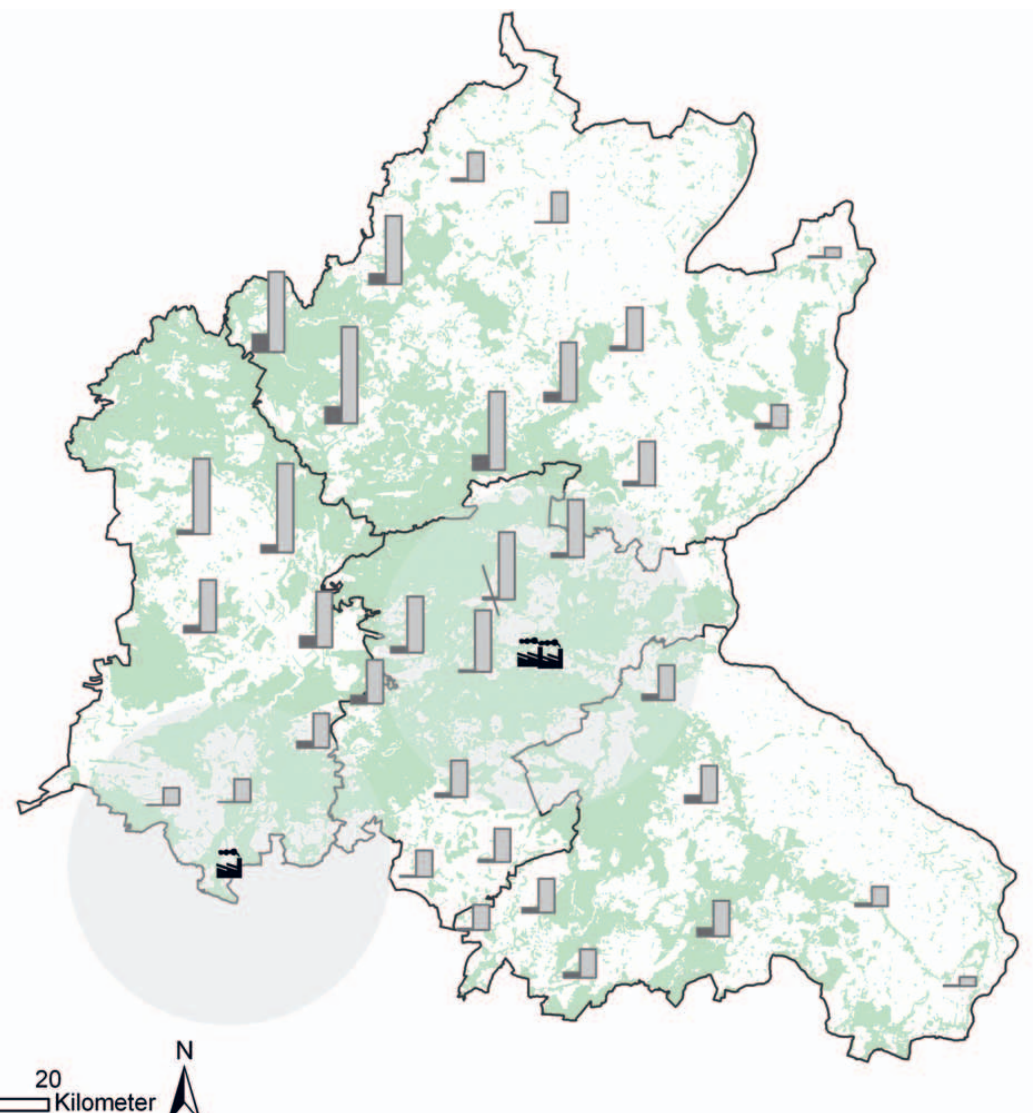

Belo-net

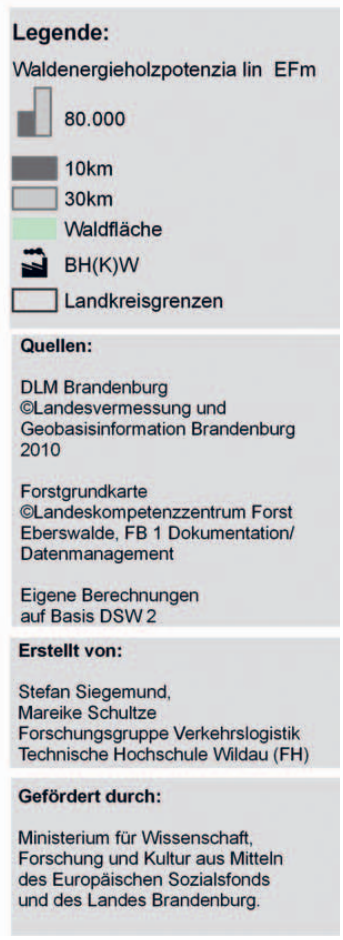

Abb. 3: Verfügbare Energieholzpotenziale (nach Szenario 3) in 10 u. 30 km Transportentfernung von den als »gut« und »sehr gut« bewerteten Standorten 
Der Kostenanteil der Vorratshaltung für die bedarfsgerechten Versorgung von Abnehmern kann bis zu $70 \%$ der Logistikkosten betragen und wird wesentlich durch die Kosten und die Auslastung der genutzten Lagerinfrastruktur beeinflusst (Schultze/Fiedler 2008). Als wesentlicher Einflussfaktor für die Möglichkeiten der Auslastung eines Biomassehofes wurden die am besten bewerteten Standorte hinsichtlich der Verfügbarkeit von Waldenergieholz in Einzugsradien von $10 \mathrm{~km}$ bzw. $30 \mathrm{~km}$ untersucht (Abb. 3). Dabei wurde der Bedarf der auf Basis von Waldenergieholz betriebenen Biomasseheiz(kraft)werke in ihren direkten Einzugsgebieten von den Potenzialdaten abgezogen.

\section{Schlussfolgerungen}

Ziel der Analyse war die Identifikation von Gebieten, die sich aufgrund der vorhandenen Rohstoff- und Infrastrukturpotenziale in besonderem Maße für den Aufbau von regionalen Wertschöpfungsketten der Energieholzbereitstellung und -nutzung eignen.

Die bestandesscharfe Modellierung von Rohholzpotenzialen ergibt unter Berücksichtigung der üblichen Verfahren der Sortimentsbildung eine jährlich verfügbare Menge an Waldenergieholz zwischen 110 und 170 Tsd t atro. Wird diese Menge lufttrocken (20\% Wassergehalt) in modernen Holzheizungen verwendet, lassen sich daraus jährlich rund 2.000 TJ Endenergie erzeugen. Mit dieser Energiemenge lassen sich ca. 30.000 Haushalte versorgen (Statistisches Bundesamt 2006) und unter Berücksichtigung aller regionalen Effekte etwa 1.000 Arbeitsplätze schaffen (Steininger et al. 2008). Berücksichtigt man die mögliche Wärmeproduktion der derzeit laufenden Biomasseheiz(kraft)werke sowie deren Nachfrage nach Waldenergieholz, lassen sich je nach Szenario 5 bis $6 \%$ des Wärmebedarfs der Region[7] aus Waldenergieholz decken.

Dabei ist zu beachten, dass die ermittelten Werte tendenziell eher eine vorsichtige Einschätzung der in den kommenden Jahren verfügbaren Energieholzmengen darstellen. So sind in der Regel die durch die Forsteinrichtung geplanten Hiebsmaßnahmen eher konservativ bemessen worden und die einbezogenen Vorräte und Zuwächse häufig höher als im DSW 2 hinterlegt. Die Bestandeswerte werden aufgrund veränderter Durchforstungskonzepte und Wuchsbedingungen (durch Nährstoffeinträge) oft unterschätzt (Offer/Staupendahl 2009). Müller (2010) stellt auf Basis von Inventurda- ten einen Anstieg der Holzvorräte in Brandenburg von 10\% zwischen den Jahren 2001 und 2008 fest.

Durch die Berechnung von drei Szenarien mit verschiedenen Verfahren der Bildung stofflicher und energetischer Holzsortimente konnten die Auswirkungen verschiedener Nutzungsentscheidungen in Abhängigkeit der Marktaktualität ermittelt werden. Bei zunehmender Konkurrenz zwischen stofflicher und energetischer Nutzung scheint das Szenario 3 am ehesten die zukünftige Entwicklung abzubilden.

Durch die Kombination der Analyse von Rohstoffpotenzialen mit einer Infrastrukturanalyse konnte eine erste Bewertung von Teilregionen hinsichtlich ihrer Eignung für den Aufbau von Biomassehöfen vorgenommen werden. Die Wahl der geeigneten Standorte basiert dabei zunächst auf allgemein verfügbaren Informationen und Geodaten. Einzelne Standorte, die z. B. durch eine Vor-Ort-Analyse und Befragung der verantwortlichen Personen näher untersucht werden müssen, werden anhand konkreter Planfälle ausgewählt werden. Um das Bewertungsmodell zu verfeinern, ist weitere Forschung zu den Einflussfaktoren auf die Wirtschaftlichkeit des Betriebs von Biomassehöfen erforderlich.

\section{Anmerkungen}

[1] Das Projekt belo-net ist ein gemeinsames Vorhaben der Hochschulen Wildau und Eberswalde. Es wird gefördert durch das Ministerium für Wissenschaft, Forschung und Kultur aus Mitteln des Europäischen Sozialfonds und des Landes Brandenburg. Weitere Informationen: www.belo-net.de

[2] G.I.S. = Geographisches Informationssystem

[3] $\mathrm{t}$ atro $=$ Tonnen Trockenmasse

[4] Der Privatwaldanteil in Nord-Ost-Brandenburg beträgt durchschnittlich 39\%. Im Landkreis Märkisch-Oderland ist diese Besitzart mit fast $60 \%$ sogar dominierend.

[5] Nadelholz: Kiefer, Fichte, Lärche, Douglasie und sonstiges Nadelholz; Laubholz: Eiche, Buche, Birke, Erle, Esche, Pappel und sonstiges Laubholz

[6] Die Verluste während einer sechsmonatigen Lagerung können je nach Lagergut und Lagerverfahren bis zu $25 \%$ betragen

[7] Bezogen auf den Wärmeverbrauch im gewerblichen und privaten Bereich, anteilig berechnet aus Daten für das Land Brandenburg uch im Bereich Wärme nach der Anzahl der Betriebe und Wohngebäude (Statistik Berlin Brandenburg 2010b und 2010c, Statistik Berlin Brandenburg 2009).

\section{Literatur}

Becker, G.; Engler, B. (2009): Stoffliche oder thermische Holznutzung: Strategien für die Sortierung von Industrie- und Kronenholz in Abhängigkeit von Bestandes- und Marktbedingungen, Vortrag auf dem Forum: Hackschnitzel - forstwirtschaftliches Produkt mit Zukunft? Vortrag auf der Ligna 2009. 
Bundesministerium für Wirtschaft und Technologie (2010): Entwicklung von Energiepreisen und Preisindizes(Deutschland) 2009; http://www.bmwi.de/BMWi/Redaktion/Binaer/ Energiedaten/ energiepreise- und-energiekosten 1 -entwicklung-energiepreise-pr eisindizes, property=blob, bereich $=$ bmwi, sprache $=\mathrm{de}, \mathrm{rwb}=$ true . xls.

Fachagentur Nachwachsende Rohstoffe (Hrsg.) (2005): Leitfaden Bioenergie, Planung, Betrieb und Wirtschaftlichkeit von Bioenergieanlagen, Gülzow.

Gothe, D.; Hahne, U. (2005): Regionale Wertschöpfung durch HolzCluster - Best-Practice-Beispiele regionaler Holz-Cluster aus den Bereichen Holzenergie, Holzhaus- und Holzmöbelbau, waldArbeitspapier Nr. 14, Freiburg: Institut für Forstbenutzung und forstliche Arbeitswissenschaft.

Haschke, P.; Rickert, I. (2002): Abschätzung der Potentiale an Waldenergieholz in den Kreisen Uckermark und Barnim, unveröffentlichte Studie, Eberswalde.

Hepperle, F. (2007): Energieholz - Konkretisierung betrieblicher Nutzungspotenziale, FVA-Einblick 3/2007, Forstliche Versuchs- und Forschungsanstalt Baden-Württemberg, Freiburg.

HNE (2010): schriftliche Mitteilungen der Arbeitsgruppe Bioenergie an der Hochschule für nachhaltige Entwicklung Eberswalde zur Datenbasis Biogasanlagen im Land Brandenburg (Stand 31.12.2009).

Hoffmann, D. (2007): Regionale Wertschöpfung durch optimierte Nutzung endogener Bioenergiepotenziale als strategischer Beitrag zur nachhaltigen Regionalentwicklung. Dissertation. Saarbrücken: Universität des Saarlandes.

Kühmaier, M., Kanzian, C., Holzleitner, F., Stampfer, K. (2007): Wertschöpfungskette Waldhackgut. Optimierung von Ernte, Transport und Logistik. Projektstudie im Auftrag von BMLFUW, Land Niederösterreich, Stadt Wien und ÖBf AG. Institut für Forsttechnik, Department für Wald und Bodenwissenschaften, Universität für Bodenkultur, Wien.

Landesamt für Umwelt, Gesundheit und Verbraucherschutz (LUGV) (2010a): Abfallaufkommen Altholz aus der Siedlungsbilanz des Landes Brandenburg im Zeitraum 2009. Landkreisweise Darstellung. Schriftliche Mitteilung des Landesamtes für Umwelt, Gesundheit und Verbraucherschutz vom 13.09.2010.

Landesamt für Umwelt, Gesundheit und Verbraucherschutz (LUGV) (2010b): Abfallaufkommen Altholz der gefährlichen Abfälle im Zeitraum 2009. Landkreisweise Darstellung des Landes Brandenburg. Schriftliche Mitteilung des Landesamtes für Umwelt, Gesundheit und Verbraucherschutz vom 16.09.2010.

Landesregierung Brandenburg (2006): Energiestrategie 2020 des Landes Brandenburg. Bericht der Landesregierung. Umsetzung des Beschlusses des Landtages, DS 4/2893-B, vom 18. Mai 2006.

Müller, Jörg (2010): Wie nachhaltig wird gewirtschaftet? - Ergebnisse der Großrauminventur 2008 auf den BWI-Punkten im Land Brandenburg, in: Ministerium für Infrastruktur und Landwirtschaft (MIL) und Landeskompentenzzentrum Forst Eberswalde (LFE) [Hrsg.]: Wissenstransfer in die Praxis. Beiträge zum 5. Winterkolloquium am 25.02.2010 in Eberswalde. Eberswalder Forstliche Schriftenreihe, Band 44, Eberswalde., 8 - 13.

Murn, Yasmin (2008): Ertragsdaten für Agrarholz, schriftliche Mitteilung im Rahmen des Projekts DENDROM - Zukunftsrohstoff Dendromasse.

Offer, A.; Staupendahl, K. (2009): Neue Bestandessortentafeln für die Waldbewertung und ihr Einsatz in der Bewertungspraxis, Forst und Holz 64, Heft 5.
Öko Institut (2004): Stoffstromanalyse zur nachhaltigen energetischen Nutzung von Biomasse. Endbericht Öko-Institut e.V, Institut Umwelt, Sicherheit, Energietechnik Umsicht, Institut für Energetik und Umwelt, ifeu, izes, Institut für Geoökologie an der Technischen Universität München.

Schneider, G.; . \& Aust, J., (2010): Schriftliche Mitteilung vom 31.08.2010 der Arbeitsgruppe Bioenergie an der Hochschule für nachhaltige Entwicklung basierend auf Daten der 50Hertz Transmission GmbH 2010 und Bilke et al. (2007).

Schubert, D. (2007): Regionale Wertschöpfungs(ketten)partnerschaften (RWP) in der ländlichen Entwicklung. Hintergrund, Ziele, Steuerung und Potenziale, Fachpapier im Rahmen der Begleitforschung von Regionen Aktiv.

Schultze, M.; Fiedler, P. (2008): Modelle für die logistische Bereitstellung von Dendromasse auf regionaler Ebene, in: Murach, D.; Knur, L.; Schultze, M. (Hrsg.): DENDROM - Zukunftsrohstoff Dendromasse, Endbericht, FKZ 0330580, Verlag Kessel, Eberswalde, Berlin, Cottbus.

Statistik Berlin Brandenburg (2009): Statistisches Jahrbuch 2009. Amt für Statistik Berlin-Brandenburg. Potsdam.

Statistik Berlin Brandenburg (2010a): Statistischer Bericht A I 3 - j/09. Bevölkerung der kreisfreien Städte und Landkreise im Land Brandenburg 2009.Amt für Statistik Berlin-Brandenburg . Potsdam.

Statistik Berlin Brandenburg (2010b): Statistischer Bericht E IV 4 - j/07. Energie- und CO2-Bilanz im Land Brandenburg 2007. Hrsg. Amt für Statistik Berlin-Brandenburg. Potsdam.

Statistik Berlin Brandenburg (2010c): Statistischer Bericht F I 1 - j/09. Fortschreibung des Wohngebäude und Wohnungsbestandes im Land Brandenburg am 31. Dezember 2009. Hrsg. Amt für Statistik Berlin-Brandenburg. Potsdam.

Statistisches Bundesamt (2006): Die Nutzung von Umweltressourcen durch die Konsumaktivitäten der privaten Haushalte. Umweltökonomische Gesamtrechnung (UGR), Wiesbaden.

Steininger, K. et al. (2008): Volkswirtschaftliche Effekte einer erweiterten Biomasse-Energie-Nutzung in der Energieregion Oststeiermark, Wegener Center., Wissenschaftlicher Bericht Nr. 21-2008, Graz.

Thraen, D.; Kostmann, J. (1997): Nachhaltiges Management des Stoffstroms Holz als Bestandteil von regionaltypischen Konzepten für eine nachhaltige zukunftsfähige Entwicklung von Konversionsgebieten und strukturschwachen Regionen im Land Brandenburg.

\section{Autoren}

\section{Dipl.-Forstw. Mareike Schultze}

Forschungsgruppe Verkehrslogistik

Technische Hochschule Wildau [FH]

mareike.schultze@th-wildau.de

\section{Dipl.-Geogr. Stefan Siegemund}

Forschungsgruppe Verkehrslogistik

Technische Hochschule Wildau [FH]

stefan.siegemund@th-wildau.de

\section{Sc. Dipl. Ing. (FH) Marco Hahs}

Arbeitsgruppe Bioenergie

Hochschule für nachhaltige Entwicklung Eberswalde (FH)

Friedrich-Ebert-Str. 28, 16225 Eberswalde

marco.hahs@hnee.de 